\title{
Hermite WENO Limiting for Multi-Moment Finite-Volume Methods using the ADER-DT Time Discretization for 1-D Systems of Conservation Laws
}

\author{
Matthew R. Norman ${ }^{\mathrm{a}, *}$ \\ ${ }^{a}$ Oak Ridge National Laboratory, PO BOX 2008 MS6016, Oak Ridge, TN 37831
}

\begin{abstract}
New Hermite Weighted Essentially Non-Oscillatory (HWENO) interpolants are developed and investigated within the Multi-Moment Finite-Volume (MMFV) formulation using the ADER-DT time discretization. Whereas traditional WENO methods interpolate pointwise, function-based WENO method explicitly form a non-oscillatory, high-order polynomial over the cell in question. This study chooses a function-based approach and details how fast convergence to optimal weights for smooth flow is ensured. Methods of sixth-, eighth-, and tenth-order accuracy are developed. These are compared against traditional single-moment WENO methods of fifth-, seventh-, ninth-, and eleventh-order accuracy to compare against more familiar methods from literature. The new HWENO methods improve upon existing HWENO methods (1) by giving better resolution of unreinforced contact discontinuities and (2) by only needing a single HWENO polynomial to update both the cell mean value and cell mean derivative.

Test cases to validate and assess these methods include 1-D linear transport, the 1-D inviscid Burger's equation, and the 1-D inviscid Euler equations. Smooth and non-smooth flows are used for evaluation. These HWENO methods performed better than comparable literature-standard WENO methods for all regimes of discontinuity and smoothness in all tests herein. They exhibit improved optimal accuracy due to the use of derivatives, and they collapse to solutions similar to typical WENO methods when limiting is required. The study concludes that the new HWENO methods are robust and effective when used in the ADER-DT MMFV framework. These results are intended to demonstrate capability rather than exhaust all possible implementations.
\end{abstract}

Keywords: ADER, WENO, Hermite WENO, Differential Transform, Finite-Volume

\section{Introduction}

Systems of conservation laws govern the evolution of many important physical phenomena, and they form the core of many simulation codes. An algorithm that numerically solves a non-linear system of conservation laws must balance multiple constraints, including accuracy, robustness, runtime, and parallel efficiency. Weighted Essentially Non-Oscillatory (WENO) methods [1, 2] are good candidates in balancing these constraints because they simultaneously allow an optimal accuracy over a given stencil of cells, while providing robustness only where needed. WENO methods provide requisite damping where needed without additional parallel data transfers, which is not generally true for hyperdiffusive filters and Flux Corrected Transport. ADER time discretizations also balance these constraints well, as they require no quadrature in the spatial discretization and no steps / stages in the time discretization. Combining ADER and WENO is a natural choice because only one WENO limiting is needed per large time step, and the overhead of WENO versus traditional reconstruction is relatively smaller with ADER.

Hermite WENO (HWENO) methods [3, 4, 5, 6] use not only a stencil of values but also derivatives to reconstruct, providing a more accurate optimal reconstruction than standard WENO methods. Also, HWENO methods can perform multi-moment simulation at significantly larger time steps than Galerkin methods, and they are much more easily limited than Galerkin methods. In fact, the maximum stable CFL

* Corresponding author

Email address: normanmr@ornl.gov (Matthew R. Norman) 
of HWENO ADER methods in 1-D is unity, no matter what the order of accuracy is. The center cell's derivative comes at no cost in terms of parallel data transfer, which makes HWENO methods relatively more parallel efficient than WENO methods. Thus, ADER with HWENO seems like a natural choice in balancing the many constraints of efficient numerical integration of conservation law systems.

However, HWENO methods have suffered some difficulties in previous derivations. In [4], one must compute two separate HWENO interpolants in order to evolve the scheme, which is prohibitively expensive to be efficient in practice. The HWENO methods of this study require only one HWENO interpolant to be formed, and it updates both the cell-averaged value and cell-averaged derivative of the state. In [3], there is the problem that the method degrades to the first-order-accurate Godunov method, spatially, when discontinuities become sufficiently steep. This is not so much of a problem for reinforced discontinuities such as shocks, but for contact discontinuities and linear transport discontinuities, this will excessively diffuse discontinuities. The HWENO methods of this study perform more accurately than a first-orderaccurate Godunov at contact discontinuities, mimicking the behavior of more traditional WENO schemes. Finally, the HWENO methods herein are more similar to [3] than to [2] in the sense that they directly form full polynomials rather than point values. This provides remarkable flexibility in terms of deriving a new method, and that flexibility is demonstrated in the new methods.

First, the MMFV method and the ADER-DT time discretization will be described in detail in section 2. Then, the design philosophy of the new HWENO methods is detailed in section 3, and four HWENO methods are derived at sixth-, eighth-, and tenth-order accuracies with varying specification of low-ordered polynomials. In section 4, numerical experiments are performed to validate and assess the new HWENO methods against a baseline WENO method that is common from literature. Finally, conclusions are drawn in section 5 .

\section{Multi-Moment Finite-Volume, ADER-DT Framework}

\subsection{Multi-Moment Finite-Volume Evolution}

For the purposes of investigating new Hermite WENO reconstructions for Multi-Moment Finite-Volume methods using the ADER time discretization, this study is concerned with a generic 1-D system of conservation laws of the form:

$$
\frac{\partial \mathbf{q}}{\partial t}+\frac{\partial \mathbf{f}}{\partial x}=\mathbf{0}
$$

where $\mathbf{q}$ and $\mathbf{f}$ are vector quantities containing the conserved variables and fluxes, respectively. For the first evolution, (1) is integrated over a space-time block defined by $\Omega_{i} \times\left[t_{n}, t_{n+1}\right]$, where $\Omega_{i} \in\left[x_{i-\frac{1}{2}}, x_{i+\frac{1}{2}}\right]$, $x_{i \pm \frac{1}{2}}=x_{i} \pm \Delta x / 2, t_{n+1}=t_{n}+\Delta t$. This gives rise to:

$$
\begin{gathered}
\overline{\mathbf{q}}_{i, n+1}-\overline{\mathbf{q}}_{i, n}+\frac{\Delta t}{\Delta x}\left(\mathcal{F}_{i+\frac{1}{2}, n}-\mathcal{F}_{i-\frac{1}{2}, n}\right)=\mathbf{0} \\
\mathcal{F}_{i-\frac{1}{2}, n}=\mathcal{R}\left(\widehat{\mathbf{q}}_{i, n}\left(x_{i-\frac{1}{2}}\right), \widehat{\mathbf{q}}_{i+1, n}\left(x_{i-\frac{1}{2}}\right), \widehat{\mathbf{f}}_{i, n}\left(x_{i-\frac{1}{2}}\right), \widehat{\mathbf{f}}_{i+1, n}\left(x_{i-\frac{1}{2}}\right)\right) \\
\overline{\mathbf{q}}_{i, n}=\frac{1}{\Delta x} \int_{\Omega_{i}} \mathbf{q}_{i, n}\left(x, t_{n}\right) d \Omega \\
\widehat{\mathbf{f}}_{i, n}(x)=\frac{1}{\Delta t} \int_{t_{n}}^{t_{n+1}} \mathbf{f}_{i, n}(x, t) d t \\
\widehat{\mathbf{q}}_{i, n}(x)=\frac{1}{\Delta t} \int_{t_{n}}^{t_{n+1}} \mathbf{q}_{i, n}(x, t) d t
\end{gathered}
$$

where $\mathcal{R}$ is a flux function intended to reconcile different time-averaged fluxes sampled at the same cell interface. Note that the flux function is performed on time-averaged states and fluxes, and therefore, it should be a linear function rather than a non-linear function.

For the second evolution equations, intended to evolve the cell-averaged first-derivative, (1) is first differentiated in space and then integrated over $\Omega_{i} \times\left[t_{n}, t_{n+1}\right]$. This gives rise to:

$$
\overline{\mathbf{q}}_{i, n+1}^{(x)}-\overline{\mathbf{q}}_{i, n+1}^{(x)}+\frac{1}{\Delta x}\left(\mathcal{F}_{i+\frac{1}{2}, n}^{(x)}-\mathcal{F}_{i-\frac{1}{2}, n}^{(x)}\right)=\mathbf{0}
$$




$$
\begin{aligned}
& \mathcal{F}_{i-\frac{1}{2}, n}^{(x)}=\mathcal{R}\left(\widehat{\mathbf{q}}_{i, n}\left(x_{i-\frac{1}{2}}\right), \widehat{\mathbf{q}}_{i+1, n}\left(x_{i-\frac{1}{2}}\right), \widehat{\mathbf{f}}_{i, n}^{(x)}\left(x_{i-\frac{1}{2}}\right), \widehat{\mathbf{f}}_{i+1, n}^{(x)}\left(x_{i-\frac{1}{2}}\right)\right) \\
& \overline{\mathbf{q}}^{(x)}{ }_{i, n}(x)=\frac{1}{\Delta x} \int_{\Omega_{i}} \frac{\partial \mathbf{q}}{\partial x}\left(x, t_{n}\right) d \Omega \\
& \widehat{\mathbf{f}}^{(x)}{ }_{i, n}(x)=\frac{1}{\Delta t} \int_{t_{n}}^{t_{n+1}} \frac{\partial}{\partial x} \mathbf{f}_{i, n}(x, t) d t \\
& \widehat{\mathbf{q}^{(x)}}{ }_{i, n}(x)=\frac{1}{\Delta t} \int_{t_{n}}^{t_{n+1}} \frac{\partial}{\partial x} \mathbf{q}_{i, n}(x, t) d t
\end{aligned}
$$

These two evolution equations will evolve the cell-averaged values and cell-averaged spatial derivatives of $\mathbf{q}$ that are needed to form the high-order two-moment HWENO interpolants in each cell.

Three equations / equation sets will be used in this study: transport, inviscid Burger's equation, and inviscid Euler equations. The transport equation is:

$$
\frac{\partial q}{\partial t}+\frac{\partial q}{\partial x}=0
$$

The inviscid Burger's equation is:

$$
\frac{\partial q}{\partial t}+\frac{\partial q^{2} / 2}{\partial x}=0
$$

Finally, the inviscid Euler equations are:

$$
\frac{\partial}{\partial t}\left[\begin{array}{c}
\rho \\
\rho u \\
E
\end{array}\right]+\frac{\partial}{\partial x}\left[\begin{array}{c}
\rho u \\
\rho u^{2}+p \\
u(E+p)
\end{array}\right]=\left[\begin{array}{l}
0 \\
0 \\
0
\end{array}\right]
$$

where $p=(\gamma-1)\left(E-\frac{1}{2} \rho u^{2}\right)$. This study uses $\gamma=1.4$.

\subsection{Flux Function}

Assume the flux function is written as: $\mathcal{F}=\mathcal{R}\left(\mathbf{q}^{-}, \mathbf{q}^{+}, \mathbf{f}^{-}, \mathbf{f}^{+}\right)$. For the flux function, we use a characteristic, upwind, flux-based, locally frozen Riemann solver for both the value and the spatial derivative. It is valid for the derivative because it is linear, and therefore, derivatives and integrals may be moved inside the solver itself. Though this is a common flux function, the flux-based nature of it is not that common $[7,8,9,10]$. Therefore, consider a conservation law system (1). The flux divergence term can be broken into the product of the flux Jacobian, $A=\partial \mathbf{f} / \partial \mathbf{q}$, and the state spatial derivative:

$$
\frac{\partial \mathbf{q}}{\partial t}+A \frac{\partial \mathbf{q}}{\partial x}=0
$$

Then, the flux Jacobian can then be left-multiplied to the entire system, giving:

$$
A \frac{\partial \mathbf{q}}{\partial t}+A A \frac{\partial \mathbf{q}}{\partial x}=0 \Longrightarrow \frac{\partial \mathbf{f}}{\partial t}+A \frac{\partial \mathbf{f}}{\partial x}=0
$$

Now, assume the flux Jacobian is locally frozen based on the states of either side of the cell interface: $A=A\left(\mathbf{q}^{-}, \mathbf{q}^{+}\right)$. A simple average of the two states is fine. Then, the flux Jacobian is diagonalized, $A=R \Lambda R^{-1}$, where $R$ is the matrix whose columns, $\mathbf{r}_{k}$, are right eigenvectors, $\Lambda$ is the diagonal matrix of eigenvalues, and $R^{-1}$ is the matrix whose rows, $\mathbf{l}_{k}$, are left eigenvectors. Left-multiplying by left eigenvectors, $R^{-1}$, and assuming $A$ is locally constant in space and time (i.e., locally frozen) gives:

$$
\begin{gathered}
\frac{\partial \mathbf{w}}{\partial t}+\Lambda \frac{\partial \mathbf{w}}{\partial x}=0 \\
\mathbf{w}=R^{-1} \mathbf{f}
\end{gathered}
$$


Notice how the characteristic variables are built directly on the flux vector and not on the state vector. Each flux-based characteristic variable, $w_{k}$, is then taken from the upwind location as determined by the locally frozen eigenvalue for that wave.

$$
w_{k}= \begin{cases}\mathbf{l}_{k} \cdot \mathbf{f}^{-} & \text {if } \lambda_{k}>0 \\ \mathbf{l}_{k} \cdot \mathbf{f}^{+} & \text {otherwise }\end{cases}
$$

Once each characteristic variable is taken from the upwind side, the flux is reconstituted via $\mathcal{F}=R \mathbf{w}$.

For the derivative quantities, the same characteristics are used. This is valid because of the linearity of the flux function, meaning derivatives may be brought into the inner part of the solver. Derivatives are solved as:

$$
w_{k}^{(x)}= \begin{cases}\mathbf{l}_{k} \cdot \mathbf{f}^{(x)-} & \text { if } \lambda_{k}>0 \\ \mathbf{l}_{k} \cdot \mathbf{f}^{(x)+} & \text { otherwise }\end{cases}
$$

Then the flux derivative is reconstituted via $\mathcal{F}^{(x)}=R \mathbf{w}^{(x)}$

\subsection{The ADER-DT Time Discretization}

In order to solve the evolution equations (2) and (3), the functions $\mathbf{q}_{i, n}(x, t)$ and $\mathbf{f}_{i, n}(x, t)$ must be formed over each domain $\Omega_{i} \times\left[t_{n}, t_{n+1}\right]$. Fortunately, the same functions may be shared between both evolution equations, which is not always the case with HWENO implementations (see [4]). Forming the spatial interpolations using the HWENO method will be covered in section 3. For now, it is assumed that a spatial interpolation, $\mathbf{q}(x)$, is $N$ th-order-accurate, $C^{N-1}$ continuous within $\Omega_{i}$ for all $i$, and successfully yields well-defined spatial derivatives of orders $k_{x} \in[0, N-1]$ valid at each point $x_{i}$ wherein the $k_{x}$ th derivative is at least $\left(N-k_{x}\right)$ th-order-accurate.

A DT of a function is identical to a Taylor expansion coefficient of that function, and the inverse DT is the Taylor series itself.

$$
\mathbf{Q}\left(k_{x}, k_{t}\right)=\left.\frac{1}{k_{x} ! k_{t} !} \frac{\partial^{k_{x}+k_{t}} \mathbf{q}(x, t)}{\partial x^{k_{x}} \partial t^{k_{t}}}\right|_{x=x_{0}, t=t_{n}} \quad ; \quad \mathbf{q}(x, t)=\sum_{k_{t}=0}^{N-1} \sum_{k_{x}=0}^{N-1-k_{t}} \mathbf{Q}\left(k_{x}, k_{t}\right)\left(x-x_{0}\right)^{k_{x}}\left(t-t_{n}\right)^{k_{t}}
$$

As detailed in $[7,8,11]$, DTs provide a convenient way to express the ADER philosophy of using the PDE itself to generate time and space-time information from existing spatial derivatives of the state vector, see $[12,13,14,15,16]$. The ADER-DT procedure may be used to compute $\mathbf{q}(x, t)$ and $\mathbf{f}(x, t)$ to $N$ thorder accuracy over the domain $\Omega_{i} \times\left[t_{n}, t_{n+1}\right]$. ADER-DT differs from the traditional ADER-CK (CauchyKowalewski) method in that ADER-CK uses direct symbolic expansions of the PDE, which requires costly recomputation of many terms and can be exponential in complexity in general. ADER-DT is significantly cheaper and achieves the same goal in a predictable polynomial complexity. For more information regarding these differences and other ADER-type methods, please see section 2.3.1 of [11]. Three equations will be used for evaluation in this study: 1-D linear transport, 1-D inviscid Burger's equation, and the 1-D inviscid Euler equations.

\subsubsection{Transport}

For 1-D transport, which is expressed as $\partial_{t} q+\partial_{x} q=0$, the DTs are:

$$
Q\left(k_{x}, k_{t}+1\right)=-\frac{k_{x}+1}{k_{t}+1} Q\left(k_{x}+1, k_{t}\right)
$$

The indices $k_{x}$ and $k_{t}$ represent the spatial and temporal order of a DT, respectively. Capital letters represent the DT of the a function.

\subsubsection{Burger's Equation}

For the 1-D Burger's equation, which is expressed as $\partial_{t} q+\partial_{x}\left(q^{2} / 2\right)=0$, the DTs are:

$$
\begin{gathered}
Q\left(k_{x}, k_{t}+1\right)=-\frac{k_{x}+1}{k_{t}+1} F\left(k_{x}+1, k_{t}\right) \\
F\left(k_{x}, k_{t}\right)=\frac{1}{2} \sum_{r_{t}=0}^{k_{t}} \sum_{r_{x}=0}^{k_{x}} Q\left(r_{x}, r_{t}\right) Q\left(k_{x}-r_{x}, k_{t}-r_{t}\right)
\end{gathered}
$$




\subsubsection{Euler Equations}

The 1-D Euler equations in component form are:

$$
\begin{gathered}
\frac{\partial}{\partial t}\left[\begin{array}{l}
q_{1} \\
q_{2} \\
q_{3}
\end{array}\right]+\frac{\partial}{\partial x}\left[\begin{array}{c}
q_{2} \\
q_{2} q_{2} / q_{1}+p \\
q_{2} q_{3} / q_{1}+q_{2} p / q_{1}
\end{array}\right]=\left[\begin{array}{l}
0 \\
0 \\
0
\end{array}\right] \\
p=(\gamma-1)\left(q_{3}-\frac{1}{2} q_{2} q_{2} / q_{1}\right)
\end{gathered}
$$

The DTs of the 1-D Euler equations are:

$$
\begin{gathered}
\mathbf{Q}\left(k_{x}, k_{t}+1\right)=-\frac{k_{x}+1}{k_{t}+1} \mathbf{F}\left(k_{x}+1, k_{t}\right) \\
\mathbf{F}\left(k_{x}, k_{t}\right)=\left[\begin{array}{c}
Q_{2}\left(k_{x}, k_{t}\right) \\
F_{2 a}\left(k_{x}, k_{t}\right)+P\left(k_{x}, k_{t}\right) \\
F_{3 a}\left(k_{x}, k_{t}\right)+F_{3 b}\left(k_{x}, k_{t}\right)
\end{array}\right] \\
F_{2 a}\left(k_{x}, k_{t}\right)=\frac{1}{Q_{1}(0,0)} \sum_{r_{t}=0}^{k_{t}} \sum_{r_{x}=0}^{k_{x}} Q_{2}\left(r_{x}, r_{t}\right) Q_{2}\left(k_{x}-r_{x}, k_{t}-r_{t}\right)-Q_{1}\left(r_{x}, r_{t}\right) F_{2 a}\left(k_{x}-r_{x}, k_{t}-r_{t}\right) \\
F_{3 a}\left(k_{x}, k_{t}\right)=\frac{1}{Q_{1}(0,0)} \sum_{r_{t}=0}^{k_{t}} \sum_{r_{x}=0}^{k_{x}} Q_{2}\left(r_{x}, r_{t}\right) Q_{3}\left(k_{x}-r_{x}, k_{t}-r_{t}\right)-Q_{1}\left(r_{x}, r_{t}\right) F_{3 a}\left(k_{x}-r_{x}, k_{t}-r_{t}\right) \\
P\left(k_{x}, k_{t}\right)=(\gamma-1)\left(Q_{3}\left(k_{x}, k_{t}\right)-\frac{1}{2} F_{2 a}\left(k_{x}, k_{t}\right)\right) \\
F_{3 b}\left(k_{x}, k_{t}\right)=\frac{1}{Q_{1}(0,0)} \sum_{r_{t}=0}^{k_{t}} \sum_{r_{x}=0}^{k_{x}} Q_{2}\left(r_{x}, r_{t}\right) P\left(k_{x}-r_{x}, k_{t}-r_{t}\right)-Q_{1}\left(r_{x}, r_{t}\right) F_{3 b}\left(k_{x}-r_{x}, k_{t}-r_{t}\right)
\end{gathered}
$$

\section{Hermite WENO Interpolation}

\subsection{Design Considerations}

As with any WENO method, HWENO methods rely on a linear combination of low-order-accurate polynomials to produce an interpolation that is high-order accurate when the flow is smooth, while reducing to low-order accuracy when discontinuities emerge. An optimally weighted sum of low-order-accurate polynomials will produce the exact high-order-accurate interpolation. When the flow is smooth, these optimal weights are altered little, leading to better accuracy. When the flow is non-smooth, the optimal weights are altered significantly, leading to lower accuracy but greater limiting.

There are, in general, two main types of WENO methods typically used for structured, Cartesian grids, which could be labeled as: (1) pointwise and (2) function-based.

\subsubsection{Pointwise WENO Methods}

Pointwise WENO methods are the traditional type [2], and they provide limited interpolations at a single point in space. Suppose a high-order polynomial, $P_{0}(x)$, is specified, and a series of low-order polynomials, $P_{k}(x), k \in\{1, \ldots, M\}$, are specified. The pointwise approach first determines so-called "optimal weights," $\gamma_{k}$, such that:

$$
P_{0}\left(x^{*}\right)=\sum_{k=1}^{M} \gamma_{k} P_{K}\left(x^{*}\right)
$$

where $x^{*}$ is the point at which non-oscillatory interpolation is desired. Suppose the polynomials are determined by reconstructions matrices such that $P_{k}\left(x^{*}\right)=A_{k} \vec{u}$ for $k \in\{0, \ldots, M\}$, where $\vec{u}$ is a stencil of degrees of freedom. Then, the optimal weights are found by solving:

$$
A_{0}=\sum_{k=1}^{M} \gamma_{k} A_{k}
$$


for each coefficients $\gamma_{k}$. There are times when these weights either do not exist or are poorly behaved. There are also times with some of these weights are negative, requiring special treatment [17]. Next, the pointwise approach computes so-called "limited weights," $\omega_{k}$, such that:

$$
P_{W E N O}\left(x^{*}\right)=\sum_{k=1}^{M} \omega_{k} P_{K}\left(x^{*}\right)
$$

The limited weights are constructed in such a way that $P_{W E N O}\left(x^{*}\right)$ approaches $P_{0}\left(x^{*}\right)$ when the flow is smooth but favors one or more of the lower-ordered polynomial estimates when discontinuities exist, so as to avoid the discontinuity.

Pointwise WENO methods may be used with ADER methods via the sub-cell method [18], wherein multiple point values are computed throughout the domain and then used to reconstruct a limited high-order polynomial. This method worked well in [11] because the optimal weights were well behaved when sampling at Gauss-Legendre-Lobatto (GLL) points within the domain. In fact, the optimal weights were all positive. However, it has been discovered via experimentation that weights are not always so well-behaved with many HWENO methods. Thus, significant difficulties were encountered when trying to use the pointwise WENO approach coupled with a sub-cell technique for general HWENO methods, and the results were generally found to lack a robust stability. Also, since there are many cases for which a candidate HWENO method does not provide bounded / well-behaved optimal weights, many potential HWENO methods could not be implemented with this technique.

\subsubsection{Function-Based WENO Methods}

Function-based WENO methods directly provide a non-oscillatory function $P_{W E N O}(x)$, rather than a single point estimate. A function-based WENO method also begins by specifying an optimal-order-accurate polynomial, $P_{0}(x)$, along with a set of $M$ lower-order-accurate polynomials, $P_{k}(x), k \in\{1, \ldots, M\}$. Unlike pointwise WENO methods, however, function-based WENO methods actually compute $P_{0}(x)$ during runtime. Then, a set of weights, $\gamma_{k}$ or $k \in\{1, \ldots, M\}$ and $\gamma_{B}$, is determined by the user such that:

$$
P_{0}(x)=\sum_{k=1}^{M} \gamma_{k} P_{k}(x)+\gamma_{B} P_{B}(x)
$$

where $P_{B}$ is a polynomial that exists solely to enable the WENO interpolant to approach the optimal interpolant when the flow is smooth. This study terms it the "bridge" polynomial. Note that although $P_{B}$ contains the same number of terms as $P_{0}$, it has the same order of accuracy as the lower-order-accurate polynomials, $P_{1}$ through $P_{M}$. $P_{B}$ is not directly computed but is solved for:

$$
P_{B}(x)=\frac{1}{\gamma_{B}}\left(P_{0}(x)-\sum_{k=1}^{M} \gamma_{k} P_{k}(x)\right)
$$

Then, the final WENO polynomial is produced by transforming the optimal weights, $\gamma_{k}$, into limited weights, $\omega_{k}$ :

$$
P_{W E N O}(x)=\sum_{k=1}^{M} \omega_{k} P_{k}(x)+\omega_{B} P_{B}(x)
$$

Notice that function-based WENO methods are not only valid at a single point but provide a limited function that is valid over the entire cell domain. This circumvents the need for a sub-cell-type treatment. Also, it is considerably more flexible and easier to develop than the pointwise WENO method for several reasons. First, whereas pointwise WENO methods must construct and solve a linear system (that must be well-behaved) to obtain optimal weights, function-based WENO methods can specify any set of low-order polynomials and any optimal-order polynomial with the constraint that they are all valid over the center cell domain. In fact, the union of the domains of the low-order polynomials does not even have to span the domain of the optimal-order polynomial, and the scheme will still be valid. Similarly, the optimal polynomial does not have to span the union of the lower-ordered polynomial stencils. Second, the user chooses the weights, providing flexibility to favor certain polynomials and improve overall behavior as the data becomes more 
discontinuous. This also avoids any need for treating negative weights. Finally, a sub-cell procedure is not necessary, meaning one does not have to sample at a set of GLL points, hoping the optimal weights are well-behaved at those points, and then reconstruct.

That being said, the primary disadvantage of the function-based approach, as compared to the pointwise approach, is that total variation must be computed for the bridge polynomial, which is typically more oscillatory than the lower-ordered polynomials because it contains derivatives from the optimal polynomial. This leads to difficulty in getting the limited weights to converge quickly to optimal weights as the flow becomes smooth. This can be successfully treated, however, with some additional treatment applied to the total variation estimates. Also, note that the direct computation of the optimal-ordered polynomial is not a disadvantage computationally, at least for ADER methods in particular, because this is the same expense as reconstructing an optimal-order-accurate polynomial from point values using the sub-cell technique. The computation of total variation for the bridge polynomial, however, is an additional expense. Compared to the cost of the ADER-DT procedure, however, it is not significant.

The flexibility and ease of developing a robust method that comes with function-based WENO methods leads to them being chosen for implementation in this study. Difficulty converging as the flow becomes smoother will be addressed in section 3.3.2.

\subsubsection{Improvements to Existing HWENO Schemes}

With the HWENO method of [4], one must actually create two HWENO polynomials. The polynomial used for sampling the state value is fifth-order-accurate, neglecting the center cell's derivative, and the polynomial used for the state derivative is sixth-order-accurate, including the center cell's derivative. As mentioned in that study, the primary reason for this was that the fifth-order-accurate HWENO construction did not yield bounded optimal weights for the state derivative at cell boundaries. This is a disadvantage in terms of runtime for their method, but it would be quite debilitating for an ADER method, which cannot afford to compute time derivatives twice per cell per time step. This study avoids such problems by using the function-based approach, which always has well-behaved optimal weights because they are chosen a priori completely at the user's discretion. Thus, the same HWENO interpolant is always used to update both the cell-averaged state values and the cell-averaged state derivatives, requiring only one ADER-DT procedure per cell per time step.

Also, the HWENO method of [3] suffers severe inaccuracies at contact discontinuities, which would lead to poor performance for flows in the subsonic regime. That problem is avoided here by using a more traditional choice of stencils, which, in all experiments herein, still provide well-limited simulations for shocks, contact discontinuities, and rarefactions. Accuracy at strong discontinuities will be shown to be similar to or better than traditional pointwise WENO methods.

\subsubsection{Other Considerations}

It was noticed through experimentation that when an HWENO method does not include completely one-sided stencils without derivatives, pronounced overshoots and undershoots can still occur. These onesided stencils increase the halo region of an HWENO scheme, but they can also increase the optimal order of interpolation, giving an accuracy benefit. Another observation was that the lower-ordered polynomials should be of the same order and be based on the same number of degrees of freedom to help weights converge to optimal weights better as the flow becomes smoother.

The center cell's derivative is quite valuable in terms of accuracy because it is closest to the cell being reconstructed and is therefore a high-quality degree of freedom to include. Also, it is the only moment besides the state value that can be included without adding to the parallel communication burden. So in terms of parallel computational efficiency and accuracy, it makes sense to try to include the center cell's derivative if at all possible.

Finally, this study found that simplicity was very helpful in forming well-behaved, accurate HWENO methods that keep optimal accuracy throughout as much of the flow regime as possible while obtaining steep yet limited estimates at strong discontinuities. Therefore, the number of lower-order-accurate polynomials is kept small. 


\subsection{Computing Limited Weights}

The limiting procedure is performed in a typical manner. The limited HWENO interpolants are given as

$$
P_{W E N O}(x)=\sum_{k=1}^{M} \omega_{k} P_{k}(x)+\omega_{B} P_{B}(x)
$$

where $\omega_{k}$ is the limited weight based on the total variation of the polynomial, $P_{k}(x)$. Limited weights are calculated based on the inverse of the cell-integrated total variation, transformed into a convex combination:

$$
\begin{aligned}
\omega_{k} & =\frac{\widehat{\omega}_{k}}{\sum_{k} \widehat{\omega}_{k}} \quad ; \quad \widehat{\omega}_{k}=\frac{\beta_{k}}{T V_{k}^{p}+\epsilon} \\
T V_{k} & =\sum_{\ell} \frac{1}{\Delta x} \int_{\Omega_{i}}\left(\frac{\partial^{\ell} P_{k}(x)}{\partial x^{\ell}}\right)^{2} \Delta x^{2 \ell} d \Omega
\end{aligned}
$$

where $p=2, k \in\{1, \ldots, M, B\}$, and $\epsilon$ is the smallest magnitude real number representable by floating point approximation (e.g., the TINY() function in FORTRAN 90). The weight mapping procedure of [19] is always applied twice to the limited weights herein (with the exception of the $\mathrm{W}^{*} 1$ methods, see section 3.3.1) for the purpose of removing the sensitivity to $\epsilon$ and helping improve convergence of the weights for smooth flows.

\subsection{Hermite WENO Method Descriptions}

The WENO methods of this study will follow the naming conventions of: $\mathrm{W} i-j-k$, where $i$ is the number of moments evolved, $j$ is the optimal order of accuracy, and $k$ is the order of accuracy of the lower-order polynomials. $\mathrm{W}^{*}$ indicates that a method is using the pointwise approach coupled with the sub-cell treatment, sampled at GLL points. The $\mathrm{W}^{*}-(2 N-1)-N$ methods serve as a point of reference for comparing the new HWENO schemes against something that is closer to existing literature. In all descriptions, $P_{0}$ is the optimal-order polynomial, and $P_{1}$ through $P_{M}$ are the low-order polynomials.

\subsection{1. $W^{*} 1-(2 N-1)-N$}

These are WENO methods following the pointwise approach and sub-cell technique, and they are commonly used in literature. They will serve as a baseline for comparison with other results. The polynomials are constrained by a stencil of cell mean values as follows:

$$
\begin{gathered}
\int_{\Omega_{i+\ell}} P_{0}(x) d \Omega=\bar{q}_{i+\ell}: \ell \in\{-N+1, \ldots, N-1\} \\
\int_{\Omega_{i+\ell}} P_{k}(x) d \Omega=\bar{q}_{i+\ell}: \ell \in\{-N+k, \ldots, k-1\}: k \in\{1, N\}
\end{gathered}
$$

Next, the methods are sampled at GLL points throughout the cell domain, similar to [11]. Finally, following the sub-cell approach, a non-oscillatory polynomial is reconstructed from the GLL points and fed into the ADER-DT procedure to complete the scheme. More details of this can be found in [11, 18].

\subsection{2. $W 2-8-3$}

This is an eighth-order-accurate HWENO method using quadratic lower-order polynomials to fall back on when discontinuities are present. It contains fully one-sided stencils without derivatives, and therefore it should be robustly limited.

$$
\begin{gathered}
\int_{\Omega_{i+\ell}} P_{0}(x) d \Omega=\bar{q}_{i+\ell}: \ell \in\{-2,-1,0,1,2\} \quad \int_{\Omega_{i+\ell}} \frac{\partial}{\partial x} P_{0}(x) d \Omega=\bar{q}^{(x)}{ }_{i+\ell}: \ell \in\{-1,0,1\} \\
\int_{\Omega_{i+\ell}} P_{1}(x) d \Omega=\bar{q}_{i+\ell}: \ell \in\{-2,-1,0\}
\end{gathered}
$$




$$
\begin{gathered}
\int_{\Omega_{i+\ell}} P_{2}(x) d \Omega=\bar{q}_{i+\ell}: \ell \in\{-1,0,1\} \\
\int_{\Omega_{i+\ell}} P_{3}(x) d \Omega=\bar{q}_{i+\ell}: \quad \ell \in\{0,1,2\}
\end{gathered}
$$

After forming these polynomials, the total variation estimates used here are as follows:

$$
\begin{gathered}
T V_{k}=\frac{13}{3} a_{3}^{2} \Delta x^{4}+a_{2}^{2} \Delta x^{2} \quad ; k \in\{1,2,3\} \\
T V_{B}=\frac{1}{16128}\left(252337135 a_{6}^{2}+24411792 a_{5} a_{7}+526470 a_{4} a_{8}\right) \Delta x^{10} \\
+\frac{1}{1120}\left(700936 a_{5}^{2}+70635 a_{4} a_{6}+1740 a_{3} a_{7}+35 a_{2} a_{8}\right) \Delta x^{8} \\
+\frac{1}{80}\left(3129 a_{4}^{2}+336 a_{3} a_{5}+10 a_{2} a_{6}\right) \Delta x^{6}+\frac{1}{6}\left(26 a_{3}^{2}+3 a_{2} a_{4}\right) \Delta x^{4}+a_{2}^{2} \Delta x^{2}
\end{gathered}
$$

where $P_{B}=\sum_{i=1}^{8} a_{i} x^{i-1}$ and $P_{k}=\sum_{i=1}^{3} a_{i} x^{i-1}$.

This HWENO method is admittedly a large departure from existing literature HWENO methods because (1) the union of the lower-order stencils does not span the optimal-order stencil, (2) it contains one extra cell mean value at either end of the traditional 6th-order-accurate HWENO stencil, and (3) there are no directionally-biased cubic polynomials to fall back on when the optimal stencil becomes too oscillatory. Instead it jumps directly to quadratic polynomials similar to the $\mathrm{W}^{*} 1-5-3$ scheme. Differences (1) and (3) were exercised here because it was discovered that simplicity made it significantly easier to construct a function-based HWENO method that is optimal as long as possible and then collapses to a well-behaved limiting that is similar to existing single-moment WENO methods. Difference (2) was exercised to improve the behavior of HWENO when limiting is necessary.

The design philosophy of this method was (1) to maintain optimal accuracy for as much of the flow as possible without introducing oscillations and (2) to match the accuracy of $\mathrm{W}^{*} 1-5-3$ when limiting must occur. With these goals in mind, two mechanisms were used to control the convergence of W2-8-3 as the flow became increasingly discontinuous. The first mechanism controls convergence for flows at the smoother end of the spectrum, wherein $T V_{1}, T V_{2}$, and $T V_{3}$ are almost equal. $T V_{B}$ is "handicapped" to keep it similar to the lower-order TV estimates. This handicapping is performed as follows:

$$
T V_{B}^{*}=T V_{B}+\left(\frac{1}{M} \sum_{k=1}^{M} T V_{k}-T V_{B}\right) \sigma
$$

If $\sigma=1$, then $T V_{B}$ will be equal to the average TV of the lower-ordered polynomials. If $\sigma=0$, then $T V_{B}$ remains unchanged. Determining $\sigma$ is a balance. If it is too small, then the HWENO interpolant will not converge for smoother flows. If it is too large, then (1) the HWENO polynomial will oscillate too much and (2) the bridge polynomial will not fall out when it needs to and will interfere with the accuracy of the lower-ordered polynomials in extreme limiting cases.

The second mechanism is intended to begin taking effect when $T V_{B}^{*}$ has become much larger than $T V_{1}$, $T V_{2}$, and $T V_{3}$. The optimal weight, $\gamma_{2}$, is set such that the center quadratic polynomial, $P_{2}(x)$, is given more weight than its one-sided neighbors. For simplicity, the optimal weights are computed as $\gamma_{k}=\widehat{\gamma}_{k} / \sum_{k} \widehat{\gamma}_{k}$ where $k \in\{1, \ldots, M, B\}$ to ensure convex weights, and only $\widehat{\gamma}_{k}$ are explicitly set. In this study, $\widehat{\gamma}_{1}, \widehat{\gamma}_{3}$, and $\widehat{\gamma}_{B}$ are set to unity, and $\widehat{\gamma}_{2}$ is tuned using experimentation to give good convergence once the optimal polynomial is no longer being used.

Through experimentation and simple grid searches for $\sigma$ and $\widehat{\gamma}_{2}$, particularly from experiments in section 4.2 , it was found that $\sigma=0.97$ and $\widehat{\gamma}_{2}=5$ gave good overall results for W2-8-3. This study does not claim optimality for these parameters, as optimality must define a cost function that includes a range of data steepness to ensure the HWENO polynomial is properly convergent at all regimes of flow. Such work is considered beyond the scope of this study. Also note that good values of $\sigma$ and $\widehat{\gamma}_{2}$ will depend heavily on the choice of the TV estimate. 


\subsubsection{W2-10-4}

This is a two-moment, tenth-order-accurate WENO method using cubic lower-order polynomials to fall back on when discontinuities are present.

$$
\begin{gathered}
\int_{\Omega_{i+\ell}} P_{0}(x) d \Omega=\bar{q}_{i+\ell}: \ell \in\{-3,-2,-1,0,1,2,3\} \quad \int_{\Omega_{i+\ell}} \frac{\partial}{\partial x} P_{0}(x) d \Omega=\bar{q}^{(x)}{ }_{i+\ell}: \ell \in\{-1,0,1\} \\
\int_{\Omega_{i+\ell}} P_{1}(x) d \Omega=\bar{q}_{i+\ell}: \ell \in\{-3,-2,-1,0\} \\
\int_{\Omega_{i+\ell}} P_{2}(x) d \Omega=\bar{q}_{i+\ell}: \ell \in\{-2,-1,0,1\} \\
\int_{\Omega_{i+\ell}} P_{3}(x) d \Omega=\bar{q}_{i+\ell}: \ell \in\{-1,0,1,2\} \\
\int_{\Omega_{i+\ell}} P_{4}(x) d \Omega=\bar{q}_{i+\ell}: \ell \in\{0,1,2,3\}
\end{gathered}
$$

The design philosophy of this method was (1) to maintain optimal accuracy for as much of the flow as possible without introducing oscillations and (2) to match the accuracy of $\mathrm{W}^{*} 1-7-4$ when limiting must occur. The same mechanisms as before are used here as well. Here, $\widehat{\gamma}_{1}=\widehat{\gamma}_{4}=\widehat{\gamma}_{B}=1$, and then $\widehat{\gamma}_{2}=\widehat{\gamma}_{3}$ is tuned for convergence. For this method, it was determined that $\sigma=0.999$ and $\widehat{\gamma}_{2}=\widehat{\gamma}_{3}=17$ gave good convergence. The total variation estimates used here are as follows:

$$
\begin{aligned}
& T V_{k}=\frac{3129}{80} a_{4}^{2} \Delta x^{6}+\frac{1}{6}\left(26 a_{3}^{2}+3 a_{2} a_{4}\right) \Delta x^{4}+a_{2}^{2} \Delta x^{2} \quad ; k \in\{1,2,3,4\} \\
T V_{B}= & \frac{1}{16128}\left(126 a_{10} a_{2}+252337135 a_{6}^{2}+24411792 a_{5} a_{7}+526470 a_{4} a_{8}+8288 a_{3} a_{9}\right) \Delta x^{10} \\
& +\frac{1}{1120}\left(700936 a_{5}^{2}+70635 a_{4} a_{6}+1740 a_{3} a_{7}+35 a_{2} a_{8}\right) \Delta x^{8} \\
& +\frac{1}{80}\left(3129 a_{4}^{2}+336 a_{3} a_{5}+10 a_{2} a_{6}\right) \Delta x^{6}+\frac{1}{6}\left(26 a_{3}^{2}+3 a_{2} a_{4}\right) \Delta x^{4}+a_{2}^{2} \Delta x^{2}
\end{aligned}
$$

where $P_{B}=\sum_{i=1}^{10} a_{i} x^{i-1}$ and $P_{k}=\sum_{i=1}^{4} a_{i} x^{i-1}$.

\subsubsection{W2-10-3}

This is a two-moment, tenth-order-accurate WENO method using quadratic lower-order polynomials to fall back on when discontinuities are present.

$$
\begin{gathered}
\int_{\Omega_{i+\ell}} P_{0}(x) d \Omega=\bar{q}_{i+\ell}: \ell \in\{-2,-1,0,1,2\} \quad \int_{\Omega_{i+\ell}} \frac{\partial}{\partial x} P_{0}(x) d \Omega=\bar{q}^{(x)}{ }_{i+\ell}: \ell \in\{-2,-1,0,1,2\} \\
\int_{\Omega_{i+\ell}} P_{1}(x) d \Omega=\bar{q}_{i+\ell}: \ell \in\{-2,-1,0\} \\
\int_{\Omega_{i+\ell}} P_{2}(x) d \Omega=\bar{q}_{i+\ell}: \ell \in\{-1,0,1\} \\
\int_{\Omega_{i+\ell}} P_{3}(x) d \Omega=\bar{q}_{i+\ell}: \ell \in\{0,1,2\}
\end{gathered}
$$

After forming these polynomials, the total variation estimates used here are as follows:

$$
T V_{k}=\frac{13}{3} a_{3}^{2} \Delta x^{4}+a_{2}^{2} \Delta x^{2} \quad ; k \in\{1,2,3\}
$$




$$
\begin{aligned}
T V_{B}= & \frac{1}{16128}\left(126 a_{10} a_{2}+252337135 a_{6}^{2}+24411792 a_{5} a_{7}+526470 a_{4} a_{8}+8288 a_{3} a_{9}\right) \Delta x^{10} \\
& +\frac{1}{1120}\left(700936 a_{5}^{2}+70635 a_{4} a_{6}+1740 a_{3} a_{7}+35 a_{2} a_{8}\right) \Delta x^{8} \\
& +\frac{1}{80}\left(3129 a_{4}^{2}+336 a_{3} a_{5}+10 a_{2} a_{6}\right) \Delta x^{6}+\frac{1}{6}\left(26 a_{3}^{2}+3 a_{2} a_{4}\right) \Delta x^{4}+a_{2}^{2} \Delta x^{2}
\end{aligned}
$$

where $P_{B}=\sum_{i=1}^{10} a_{i} x^{i-1}$ and $P_{k}=\sum_{i=1}^{3} a_{i} x^{i-1}$. For this method, $\sigma=0.988, \widehat{\gamma}_{1}=\widehat{\gamma}_{3}=\widehat{\gamma}_{B}=1$, and $\widehat{\gamma}_{2}=5$ were used.

\subsubsection{W2-6-3}

This is a two-moment, sixth-order-accurate WENO method intended to demonstrate the flexibility of the function-based approach in that the low-order polynomials can actually include degrees of freedom that the high-order polynomial does not include. For instance, suppose one wishes to have a sixth-order-accurate method using the cell-averaged values and derivatives over a 3-cell stencil, that collapses to something similar to a $\mathrm{W}^{*} 1-5-3$ method when limited. In that case, the following method would work well. This would provide good accuracy for non-smooth flow, while not requiring the expense of eighth-order accuracy overall if the user deems that too large.

$$
\begin{gathered}
\int_{\Omega_{i+\ell}} P_{0}(x) d \Omega=\bar{q}_{i+\ell}: \ell \in\{-1,0,1\} \quad \int_{\Omega_{i+\ell}} \frac{\partial}{\partial x} P_{0}(x) d \Omega=\overline{q^{(x)}}{ }_{i+\ell}: \ell \in\{-1,0,1\} \\
\int_{\Omega_{i+\ell}} P_{1}(x) d \Omega=\bar{q}_{i+\ell}: \ell \in\{-2,-1,0\} \\
\int_{\Omega_{i+\ell}} P_{2}(x) d \Omega=\bar{q}_{i+\ell}: \ell \in\{-1,0,1\} \\
\int_{\Omega_{i+\ell}} P_{3}(x) d \Omega=\bar{q}_{i+\ell}: \ell \in\{0,1,2\}
\end{gathered}
$$

After forming these polynomials, the total variation estimates used here are as follows:

$$
\begin{gathered}
T V_{k}=\frac{13}{3} a_{3}^{2} \Delta x^{4}+a_{2}^{2} \Delta x^{2} \quad ; k \in\{1,2,3\} \\
T V_{B}=\frac{252337135}{16128} a_{6}^{2} \mathrm{dx}{ }^{10}+\frac{1}{1120}\left(700936 a_{5}^{2}+70635 a_{4} a_{6}\right) \mathrm{dx}^{8} \\
+\frac{1}{80}\left(3129 a_{4}^{2}+336 a_{3} a_{5}+10 a_{2} a_{6}\right) \mathrm{dx}^{6}+\frac{1}{6}\left(26 a_{3}^{2}+3 a_{2} a_{4}\right) \mathrm{dx}+a_{2}^{2} \mathrm{dx}^{2}
\end{gathered}
$$

where $P_{B}=\sum_{i=1}^{6} a_{i} x^{i-1}$ and $P_{k}=\sum_{i=1}^{3} a_{i} x^{i-1}$. For this method, $\sigma=0.9, \widehat{\gamma}_{1}=\widehat{\gamma}_{3}=\widehat{\gamma}_{B}=1$, and $\widehat{\gamma}_{2}=5$ were used.

\section{Numerical Experiments}

All experiments except section 4.4.1 are run on the domain [0,1], and cell averages are initialized with high-order-accurate quadrature. All simulations use a CFL value of 0.9 .

\subsection{Transport of a Sine Wave}

A single-period sine wave defined by $q(x, 0)=(\sin (2 \pi x)+1) / 2$ is transported in 1-D in order to determine schemes' convergence behavior for smooth flows. Since the WENO error norms only differed from the unlimited error norms by a relative magnitude of $10^{-6}$ or less, as expected for very smooth flows, the convergences are reported once to avoid redundancy. Table 1 demonstrates the expected convergence rates for the HWENO methods developed herein both in the $L_{1}$ and $L_{\infty}$ error norms. 


\begin{tabular}{|c|c||c|c|c|c|}
\hline \multicolumn{2}{|c|}{$\mathrm{nx}$} & 15 & 20 & 25 & 30 \\
\hline \hline \multirow{4}{*}{ W2-6-3 } & $L_{1}$ & $2.50 \mathrm{E}-06$ & $5.03 \mathrm{E}-07$ & $1.35 \mathrm{E}-07$ & $4.53 \mathrm{E}-08$ \\
\cline { 2 - 6 } & ord & & 5.57 & 5.88 & 6.00 \\
\cline { 2 - 6 } & $L_{\infty}$ & $3.91 \mathrm{E}-06$ & $7.89 \mathrm{E}-07$ & $2.13 \mathrm{E}-07$ & $7.11 \mathrm{E}-08$ \\
\cline { 2 - 6 } & ord & & 5.57 & 5.87 & 6.01 \\
\hline \multirow{4}{*}{ W2-8-3 } & $L_{1}$ & $3.33 \mathrm{E}-08$ & $3.74 \mathrm{E}-09$ & $6.44 \mathrm{E}-10$ & $1.50 \mathrm{E}-10$ \\
\cline { 2 - 6 } & ord & & 7.60 & 7.89 & 8.00 \\
\cline { 2 - 6 } & $L_{\infty}$ & $5.23 \mathrm{E}-08$ & $5.86 \mathrm{E}-09$ & $1.01 \mathrm{E}-09$ & $2.35 \mathrm{E}-10$ \\
\cline { 2 - 6 } & ord & & 7.61 & 7.88 & 8.00 \\
\hline & $L_{1}$ & $5.95 \mathrm{E}-10$ & $3.77 \mathrm{E}-11$ & $4.14 \mathrm{E}-12$ & $6.67 \mathrm{E}-13$ \\
\cline { 2 - 6 } & ord & & 9.59 & 9.91 & 10.01 \\
\cline { 2 - 6 } & $L_{\infty}$ & $9.33 \mathrm{E}-10$ & $5.83 \mathrm{E}-11$ & $6.49 \mathrm{E}-12$ & $1.05 \mathrm{E}-12$ \\
\cline { 2 - 6 } & ord & & 9.64 & 9.84 & 10.02 \\
\hline & $L_{1}$ & $3.83 \mathrm{E}-10$ & $1.70 \mathrm{E}-11$ & $1.85 \mathrm{E}-12$ & $2.93 \mathrm{E}-13$ \\
\cline { 2 - 6 } W2-10-3 & ord & & 10.83 & 9.94 & 10.10 \\
\cline { 2 - 6 } & $L_{\infty}$ & $9.61 \mathrm{E}-10$ & $2.68 \mathrm{E}-11$ & $2.90 \mathrm{E}-12$ & $4.61 \mathrm{E}-13$ \\
\cline { 2 - 6 } & ord & & 12.44 & 9.96 & 10.10 \\
\hline
\end{tabular}

Table 1: Error Norms and grid convergence rates for the HWENO methods, transporting a single-period sine wave over the domain 3 times.

\begin{tabular}{|c|c|}
\hline Method & CPU Time (s) \\
\hline \hline W2-10-4 & 7.53151 \\
\hline W2-10-3 & 5.52718 \\
\hline W2-8-3 & 4.85065 \\
\hline W2-6-3 & 4.30781 \\
\hline
\end{tabular}

Table 2: CPU times for $10^{7}$ invocations of the W2 HWENO reconstructions for a single cell.

To give the reader a sense of comparative cost between the W2 methods, Table 2 lists the CPU runtimes for $10^{7}$ invocations of each of the W2 methods. Please keep in mind the following, however, when using these runtimes for decision making purposes. For systems of equations, some HWENO routines apply to all variables and some routines apply only to one (e.g., often times, only density forms non-linear weights for euler equations). This affects runtimes for HWENO reconstruction in practice because the non-linear weights and mapping are a substantial cost. These are only in 1-D, and in multiple dimensions, reconstruction generally costs $\mathcal{O}\left(N^{D+1}\right)$, whereas error still convergense at $\mathcal{O}\left(\Delta x^{N}\right)$. So efficiency will differ in multiple dimensions. Also, HWENO cost within the overall code depends on dimensionality, optimal accuracy, and complexity / nonlinearity. For a non-linear function, DTs require $\mathcal{O}\left(N^{2(D+1)}\right)$ operations while HWENO $\operatorname{costs} \mathcal{O}\left(N^{D+1}\right)$. With higher dimensionality and complexity, computing DTs dominates overall cost, and HWENO becomes a small overhead, though this would be different with another time integrator. Finally, smoothness heavily affects accuracy and thus efficiency for an HWENO method. Also, the reader may refer to [20] for another WENO ADER runtime comparison and to [21] for some rough notions of computational complexity for another WENO ADER scheme.

\subsection{Transport of a tanh-based Function}

It is beneficial to gain a sense of how WENO functions behave, not just over a strong discontinuity, but over a range of functions with steadily increasing non-smoothness. To this end, the following function is used with transport: $q(x, 0)=\frac{1}{2}\left(\tanh \left[\left(x-x_{1}\right) m\right]-\tanh \left[\left(x-x_{2}\right) m\right]\right)$, where $x_{1}=0.4, x_{2}=0.6$, and $m \in(0, \infty)$ is a "steepness parameter", which renders the function more discontinuous as $m$ increases. The function is evolved starting with $m=30$ at the smoothest and $m=10^{6}$ at the steepest, and 400 cells are used in each run. At $m=10^{6}$, the tanh-based function is indistinguishable from the so-called "square wave" when run on 400 cells. The reason for starting at $m=30$ and using 400 cells is twofold. First, the discontinuity in derivatives at the periodic boundary is rendered sufficiently small that it does not affect results, and this 


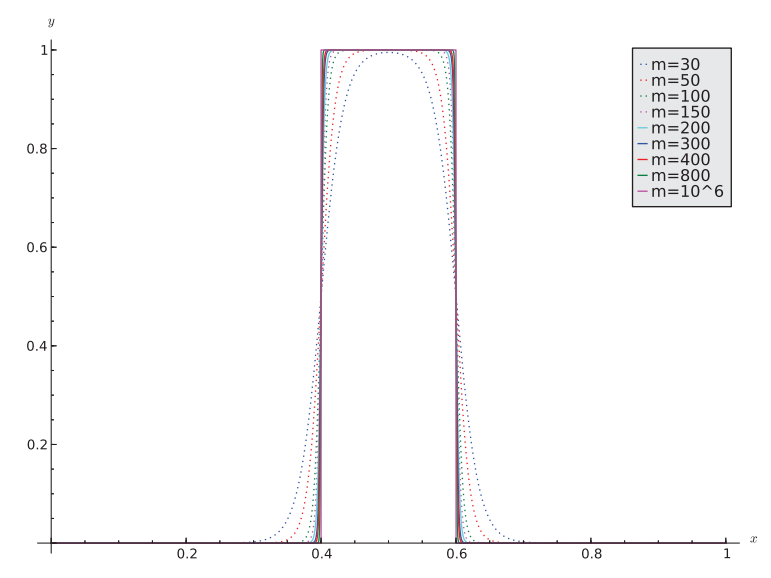

(a) Plot over the full domain.

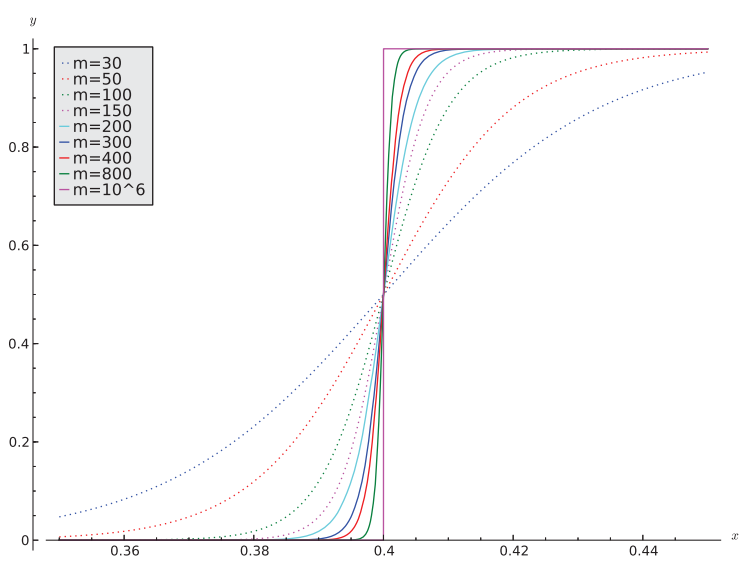

(b) Zoomed in at the discontinuity.

Figure 1: Plots of the tanh-based functions over a range of $m$.

\begin{tabular}{|c||c|c|c|c|c|c|c|c|}
\hline $\mathrm{m}$ & W2-6-3 & W2-8-3 & W2-10-4 & W2-10-3 & $\mathrm{W}^{*} 1-5-3$ & $\mathrm{~W}^{*} 1-7-4$ & $\mathrm{~W}^{*} 1-9-5$ & $\mathrm{~W}^{*} 1-11-6$ \\
\hline \hline 30 & $1.42 \mathrm{E}-08$ & $1.30 \mathrm{E}-10$ & $2.75 \mathrm{E}-12$ & $1.22 \mathrm{E}-12$ & $8.03 \mathrm{E}-07$ & $1.86 \mathrm{E}-08$ & $7.27 \mathrm{E}-10$ & $4.27 \mathrm{E}-11$ \\
\hline 50 & $2.69 \mathrm{E}-07$ & $6.62 \mathrm{E}-09$ & $3.58 \mathrm{E}-10$ & $1.62 \mathrm{E}-10$ & $9.89 \mathrm{E}-06$ & $6.11 \mathrm{E}-07$ & $6.18 \mathrm{E}-08$ & $9.24 \mathrm{E}-09$ \\
\hline 100 & $1.10 \mathrm{E}-05$ & $1.02 \mathrm{E}-06$ & $1.94 \mathrm{E}-07$ & $9.73 \mathrm{E}-08$ & $2.11 \mathrm{E}-04$ & $4.54 \mathrm{E}-05$ & $1.39 \mathrm{E}-05$ & $5.51 \mathrm{E}-06$ \\
\hline 150 & $9.06 \mathrm{E}-05$ & $2.34 \mathrm{E}-05$ & $4.21 \mathrm{E}-06$ & $1.28 \mathrm{E}-05$ & $4.55 \mathrm{E}-04$ & $2.67 \mathrm{E}-04$ & $1.22 \mathrm{E}-04$ & $7.50 \mathrm{E}-05$ \\
\hline 200 & $4.52 \mathrm{E}-04$ & $3.98 \mathrm{E}-04$ & $2.52 \mathrm{E}-05$ & $4.96 \mathrm{E}-04$ & $7.10 \mathrm{E}-04$ & $4.02 \mathrm{E}-04$ & $3.07 \mathrm{E}-04$ & $2.74 \mathrm{E}-04$ \\
\hline 300 & $2.19 \mathrm{E}-03$ & $2.20 \mathrm{E}-03$ & $7.55 \mathrm{E}-04$ & $2.18 \mathrm{E}-03$ & $2.22 \mathrm{E}-03$ & $8.40 \mathrm{E}-04$ & $4.99 \mathrm{E}-04$ & $4.46 \mathrm{E}-04$ \\
\hline 400 & $3.20 \mathrm{E}-03$ & $3.21 \mathrm{E}-03$ & $1.48 \mathrm{E}-03$ & $3.21 \mathrm{E}-03$ & $3.30 \mathrm{E}-03$ & $1.76 \mathrm{E}-03$ & $1.11 \mathrm{E}-03$ & $8.24 \mathrm{E}-04$ \\
\hline 800 & $4.92 \mathrm{E}-03$ & $4.92 \mathrm{E}-03$ & $3.28 \mathrm{E}-03$ & $4.93 \mathrm{E}-03$ & $5.01 \mathrm{E}-03$ & $3.45 \mathrm{E}-03$ & $2.77 \mathrm{E}-03$ & $2.37 \mathrm{E}-03$ \\
\hline $1.00 \mathrm{E}+06$ & $6.65 \mathrm{E}-03$ & $6.65 \mathrm{E}-03$ & $5.02 \mathrm{E}-03$ & $6.66 \mathrm{E}-03$ & $6.74 \mathrm{E}-03$ & $5.18 \mathrm{E}-03$ & $4.50 \mathrm{E}-03$ & $4.10 \mathrm{E}-03$ \\
\hline
\end{tabular}

Table 3: $L_{1}$ errors for the tanh-based function over a range of the steepness parameter $m$.

violation of periodicity becomes successively smaller as $m$ increases. Second, the function itself is resolved well enough at 400 cells that it is able to converge at greater than tenth-order, meaning it is "smooth" at $m=30$. This is also supported by the observation that WENO and optimal errors norms are the same at $m=30$. The steepnesses used are $m=30,50,100,150,200,300,400,800,10^{6}$. These functions are plotted in Figure 1.

In Table 3 , the $L_{1}$ errors for each of the WENO and HWENO methods are tabulated over a range of the steepness parameter, $m$, in order to get a better handle on how each of these methods perform over a range of smoothness. These experiments were used to determine decent values of $\sigma$ and optimal weights in sections 3.3.2-3.3.5. The goal was to set $\sigma$ such that the optimal polynomial remains the largest influence over as much of the steepness parameter as possible, without admitting too many oscillations. Then, the goal was to set the optimal weights such that the low-order polynomials mimic the behavior of the $\mathrm{W}^{*} 1-5-3$ and $\mathrm{W}^{*} 1-7-4$ schemes.

Table 3 shows that the HWENO schemes do indeed mimic the $L_{1}$ error behavior of the single-moment WENO schemes starting around $m=200$, and the HWENO schemes are usually slightly more accurate. For the $\mathrm{W}^{*}$ schemes, using two applications of weight mapping modestly decreased the errors at the stronger discontinuities such as $m=400$ and above. However, it increased errors more at the moderate discontinuity ranges such as $m=150$ and $m=200$. For this reason, the $\mathrm{W}^{*}$ schemes only use one application of weight mapping. Comparing Table 4 against Table 3, the reader can see at which point the WENO limiting really starts to take effect for each method. One interesting difference between WENO and HWENO is that for WENO, the limited accuracy is actually better than optimal in most of the steeper cases (i.e., $m \geq 200$ ). For HWENO, however, the optimal accuracy is much better for the steeper functions, so limited accuracy typically is less than the optimal accuracy. 


\begin{tabular}{|c||c|c|c|c|c|c|c|c|}
\hline $\mathrm{m}$ & W2-6-3 & W2-8-3 & W2-10-4 & W2-10-3 & $\mathrm{W}^{*} 1-5-3$ & $\mathrm{~W}^{*} 1-7-4$ & $\mathrm{~W}^{*} 1-9-5$ & $\mathrm{~W}^{*} 1-11-6$ \\
\hline \hline 30 & $1.42 \mathrm{E}-08$ & $1.30 \mathrm{E}-10$ & $2.75 \mathrm{E}-12$ & $1.22 \mathrm{E}-12$ & $8.03 \mathrm{E}-07$ & $1.86 \mathrm{E}-08$ & $7.27 \mathrm{E}-10$ & $4.27 \mathrm{E}-11$ \\
\hline 50 & $2.69 \mathrm{E}-07$ & $6.62 \mathrm{E}-09$ & $3.58 \mathrm{E}-10$ & $1.62 \mathrm{E}-10$ & $9.89 \mathrm{E}-06$ & $6.11 \mathrm{E}-07$ & $6.18 \mathrm{E}-08$ & $9.24 \mathrm{E}-09$ \\
\hline 100 & $1.10 \mathrm{E}-05$ & $1.01 \mathrm{E}-06$ & $1.94 \mathrm{E}-07$ & $9.66 \mathrm{E}-08$ & $2.11 \mathrm{E}-04$ & $4.54 \mathrm{E}-05$ & $1.39 \mathrm{E}-05$ & $5.51 \mathrm{E}-06$ \\
\hline 150 & $7.51 \mathrm{E}-05$ & $1.43 \mathrm{E}-05$ & $4.21 \mathrm{E}-06$ & $2.36 \mathrm{E}-06$ & $7.81 \mathrm{E}-04$ & $2.77 \mathrm{E}-04$ & $1.28 \mathrm{E}-04$ & $7.22 \mathrm{E}-05$ \\
\hline 200 & $2.24 \mathrm{E}-04$ & $5.80 \mathrm{E}-05$ & $2.33 \mathrm{E}-05$ & $1.48 \mathrm{E}-05$ & $1.53 \mathrm{E}-03$ & $6.92 \mathrm{E}-04$ & $4.00 \mathrm{E}-04$ & $2.68 \mathrm{E}-04$ \\
\hline 300 & $7.26 \mathrm{E}-04$ & $2.62 \mathrm{E}-04$ & $1.53 \mathrm{E}-04$ & $1.14 \mathrm{E}-04$ & $2.92 \mathrm{E}-03$ & $1.71 \mathrm{E}-03$ & $1.19 \mathrm{E}-03$ & $9.12 \mathrm{E}-04$ \\
\hline 400 & $1.25 \mathrm{E}-03$ & $5.48 \mathrm{E}-04$ & $3.91 \mathrm{E}-04$ & $3.02 \mathrm{E}-04$ & $3.84 \mathrm{E}-03$ & $2.60 \mathrm{E}-03$ & $1.97 \mathrm{E}-03$ & $1.56 \mathrm{E}-03$ \\
\hline 800 & $2.41 \mathrm{E}-03$ & $1.72 \mathrm{E}-03$ & $1.39 \mathrm{E}-03$ & $1.23 \mathrm{E}-03$ & $5.47 \mathrm{E}-03$ & $4.37 \mathrm{E}-03$ & $3.62 \mathrm{E}-03$ & $2.98 \mathrm{E}-03$ \\
\hline $1.00 \mathrm{E}+06$ & $6.75 \mathrm{E}-03$ & $5.63 \mathrm{E}-03$ & $5.13 \mathrm{E}-03$ & $4.67 \mathrm{E}-03$ & $7.36 \mathrm{E}-03$ & $6.22 \mathrm{E}-03$ & $5.46 \mathrm{E}-03$ & $5.26 \mathrm{E}-03$ \\
\hline
\end{tabular}

Table 4: Same as Table 3 except with WENO turned off, meaning only the optimal polynomial is used.

The use of derivative moments instead of cell values only is advantageous in terms of accuracy. For instance, for $m \leq 150$, the eighth-order-accurate method is generally more accurate than even the eleventhorder-accurate method. The general rule holds that the closer the degree of freedom used for reconstruction, the more accurate the reconstruction is. Regarding the two tenth-order-accurate methods, though W2-10-3 is more accurate for smoother flows (as expected), it is not that much more accurate overall. The W2-10-4 method, however, converges much better for $m \geq 150$. Note that both W2-10-3 and W2-10-4 require the same parallel communication burden because the same volume of halo data must be communicated across a domain decomposition boundary.

\subsection{Burger's Equation}

The Burger's simulations use the same sine wave initialization of $q(x, 0)=(\sin (2 \pi x)+1) / 2$.

\subsubsection{Shock}

For the shock formation, the initial data is simulated to a time of $t=0.5$. Figure 2 plots the solution from each WENO method for the Burger's shock formation. Note that the methods are all quite similar in solution, and therefore, a zoom of the plot is given to show more clearly the differences between the methods. Most notable is that the HWENO methods all produce a sharper shock than any of the $\mathrm{W}^{*} 1$ reference methods. Also, the W2-10-4 method produces the sharpest shock of all of the methods. Each of the HWENO methods with third-order-accurate low-order polynomials gave nearly identical shocks, and this is expected since they are in the highly limited regime at that point. For the $\mathrm{W}^{*} 1$ methods, increasing the order of accuracy does not necessarily monotonically increase the sharpness of the shock solution.

\subsection{Euler Equations}

In each of the Euler equation experiments, the limited weights for density are used for each of the other variables so that each is using the same limited weight.

\subsubsection{Smooth Flow}

The smooth Euler flow is initialized with $\rho(x, 0)=1+\sin (\pi x) / 5, u(x, 0)=1$, and $p(x, 0)=1$ on a domain of $[0,2]$. Then, this is simulated out to $t=2$. The flow is smooth and therefore should produce error norms that confirm optimal accuracy for this non-linear system of equations. Table 5 gives the $L_{1}$ and $L_{\infty}$ errors for each WENO method. All methods do indeed approach the optimal order of accuracy as the grid is refined. Many, however, reach machine precision before this approach is complete.

\subsubsection{Sod Shock Tube}

The shock tube simulations are initialized with $u(x, 0)=0$ and:

$$
\rho(x, 0)= \begin{cases}1 & \text { if } x<1 / 2 \\ 0.125 & \text { otherwise }\end{cases}
$$




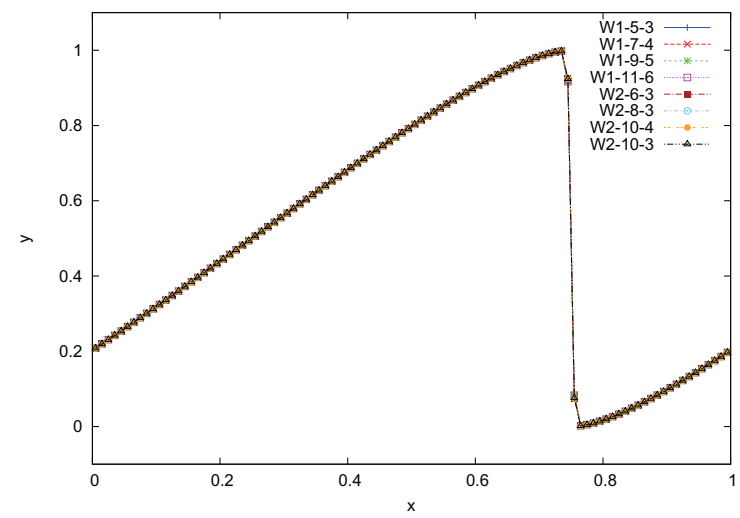

(a) Full plot of solutions from all WENO methods.

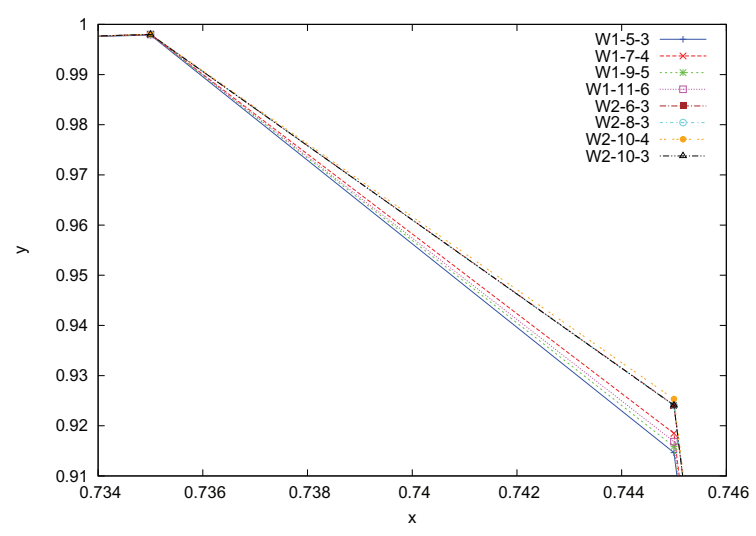

(b) Plot zoomed in at the top of the shock.

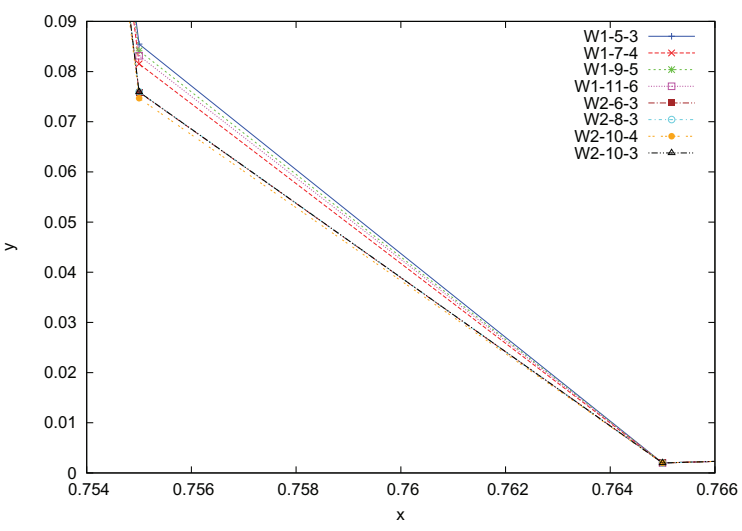

(c) Plot zoomed in at the bottom of the shock.

Figure 2: Plot of Burger's Equation shock formation solutions comparing the HWENO and WENO methods in this study.

\begin{tabular}{|c|c||c|c|c|c|c|c|}
\hline \multicolumn{2}{|c|}{$\mathrm{nx}$} & 15 & 20 & 25 & 30 & 35 & 40 \\
\hline \hline \multirow{4}{*}{ W2-6-3 } & $L_{1}$ & $3.51 \mathrm{E}-06$ & $7.34 \mathrm{E}-07$ & $2.13 \mathrm{E}-07$ & $7.65 \mathrm{E}-08$ & $3.20 \mathrm{E}-08$ & $1.49 \mathrm{E}-08$ \\
\cline { 2 - 8 } & ord & & 5.44 & 5.55 & 5.61 & 5.66 & 5.69 \\
\cline { 2 - 8 } & $L_{\infty}$ & $5.50 \mathrm{E}-06$ & $1.15 \mathrm{E}-06$ & $3.34 \mathrm{E}-07$ & $1.20 \mathrm{E}-07$ & $5.02 \mathrm{E}-08$ & $2.34 \mathrm{E}-08$ \\
\cline { 2 - 8 } & ord & & 5.45 & 5.53 & 5.62 & 5.65 & 5.72 \\
\hline \multirow{4}{*}{ W2-8-3 } & $L_{1}$ & $4.83 \mathrm{E}-08$ & $5.77 \mathrm{E}-09$ & $1.08 \mathrm{E}-09$ & $2.72 \mathrm{E}-10$ & $8.40 \mathrm{E}-11$ & $3.01 \mathrm{E}-11$ \\
\cline { 2 - 8 } & ord & & 7.39 & 7.51 & 7.57 & 7.62 & 7.67 \\
\cline { 2 - 8 } & $L_{\infty}$ & $7.58 \mathrm{E}-08$ & $8.93 \mathrm{E}-09$ & $1.69 \mathrm{E}-09$ & $4.26 \mathrm{E}-10$ & $1.32 \mathrm{E}-10$ & $4.74 \mathrm{E}-11$ \\
\cline { 2 - 8 } & ord & & 7.43 & 7.45 & 7.57 & 7.61 & 7.66 \\
\hline \multirow{4}{*}{ W2-10-4 } & $L_{1}$ & $8.87 \mathrm{E}-10$ & $6.01 \mathrm{E}-11$ & $7.23 \mathrm{E}-12$ & $1.27 \mathrm{E}-12$ & $2.90 \mathrm{E}-13$ & $8.00 \mathrm{E}-14$ \\
\cline { 2 - 8 } & ord & & 9.36 & 9.49 & 9.52 & 9.60 & 9.64 \\
\cline { 2 - 8 } & $L_{\infty}$ & $1.39 \mathrm{E}-09$ & $9.32 \mathrm{E}-11$ & $1.14 \mathrm{E}-11$ & $2.00 \mathrm{E}-12$ & $4.54 \mathrm{E}-13$ & $1.25 \mathrm{E}-13$ \\
\cline { 2 - 8 } & ord & & 9.40 & 9.43 & 9.53 & 9.61 & 9.68 \\
\hline & $L_{1}$ & $4.51 \mathrm{E}-10$ & $2.79 \mathrm{E}-11$ & $3.38 \mathrm{E}-12$ & $5.98 \mathrm{E}-13$ & $1.37 \mathrm{E}-13$ & $3.79 \mathrm{E}-14$ \\
\cline { 2 - 8 } & ord & & 9.68 & 9.46 & 9.50 & 9.56 & 9.62 \\
\cline { 2 - 8 } & $L_{\infty}$ & $7.19 \mathrm{E}-10$ & $4.35 \mathrm{E}-11$ & $5.31 \mathrm{E}-12$ & $9.37 \mathrm{E}-13$ & $2.17 \mathrm{E}-13$ & $6.08 \mathrm{E}-14$ \\
\cline { 2 - 8 } & ord & & 9.75 & 9.43 & 9.51 & 9.49 & 9.52 \\
\hline
\end{tabular}

Table 5: Error Norms and grid convergence rates for the HWENO methods, for smooth Euler flow to $t=2$. 


$$
p(x, 0)= \begin{cases}1 & \text { if } x<1 / 2 \\ 0.1 & \text { otherwise }\end{cases}
$$

on a domain of $[0,1]$.

The numerical solutions to the Sod shock tube problem are given in Figures 3 and 4, solved with 100 and 400 cells, respectively. The plots are also zoomed in on key features of the solution to show differences between the various methods. $\mathrm{W}^{*} 1-9-5$ and $\mathrm{W}^{*} 1-11-6$ are not plotted because they resulted in NaNs for a CFL of 0.9 , meaning they were too oscillatory to succeed without applying the WENO treatment to characteristics. Since the WENO treatment is applied to state variables and not characteristics, the oscillations are not surprising, but they are controlled for the methods plotted. For sake of brevity, only the density is plotted, since it contains smooth flow in the rarefaction, $C^{1}$ discontinuities at either end of the rarefaction, and two $C^{0}$ discontinuities: the shock being reinforced and the contact non-reinforced.

The two most notable differences are (1) the oscillation magnitudes about the bottom of the rarefaction and at the contact discontinuity and (2) the resolution of the rarefaction's top $C^{1}$ discontinuity. The W2-6-3, W2-8-3, and W2-10-3 methods generally exhibit the same magnitude of oscillations as the W1-5-3 scheme, which would be expected since they share the same quadratic low-ordered polynomials. Those methods oscillate relatively little. As for the W2-10-4 and $\mathrm{W}^{*} 2-7-4$ methods, they oscillate somewhat more, and given their similarity, one can likely attribute the additional oscillations to the cubic low-ordered polynomials shared between the two methods. The cubic low-ordered polynomials of W2-10-4 and $\mathrm{W}^{*} 2-7-4$ are more oscillatory than the quadratic low-ordered polynomials of W2-6-3, W2-8-3, and W2-10-3, having less inherent diffusion, and this explains the observations of oscillation magnitudes. In general, W2-10-4 exhibits slightly smaller magnitude oscillations than $\mathrm{W}^{*} 1-7-4$, and its oscillations also tail off more quickly with distance from the discontinuity than $\mathrm{W}^{*} 1-7-4$. Regarding the resolution of the discontinuity at the top of the rarefaction, the methods in order of increasing accuracy are: $\mathrm{W}^{*} 1-5-3, \mathrm{~W} 2-10-3, \mathrm{~W} 2-6-3, \mathrm{~W} 2-8-3, \mathrm{~W}^{*} 1-7-4$, and W2-10-4. In particular, having cubic lower-ordered polynomials seems to be a deciding factor in resolving this part of the flow. Finally, refining from 100 to 400 cells shows typical behavior in sharpening the resolution of every discontinuity as well as lowering the magnitude and extent of oscillations.

\section{Conclusions}

The multi-moment finite-volume, ADER-DT formulation has been detailed with a focus on the development of new Hermite WENO interpolants. Differences between the HWENO interpolants herein and those existing in literature have been given along with detailed derivations. Methods of improving convergence of limited weights toward optimal weights with increasing smoothness have been given for each method. Then, experiments were performed to validate, investigate, and compare the HWENO methods against one another and more standard WENO methods commonly used in other studies. The new HWENO methods are shown to better resolve jump discontinuities over a range of steepness than traditional WENO methods. Also, they are shown to have superior accuracy, as expected, for smooth flows due to the use of derivative information. Each proposed method reaches its optimal order of accuracy during experimentation. The new HWENO interpolants, therefore, have been shown to be effective and robust in conjunction with the multi-moment finite-volume method and the ADER-DT time discretization.

The methods implemented in this study are intended to be exploratory, not exhaustive. The intent is to guide the reader in implementing function-based HWENO methods, demonstrating a range of flexibility in this type of HWENO formulation. Future investigation on these methods will apply them to multiple dimensions, new equation sets, and new scientific applications. It is worth noting that these HWENO methods are not confined only to ADER-DT time discretizations but are valid for any ADER time discretization. They are also applicable even to ODE solver-based time discretizations such as Runge-Kutta as well as other ADER-type discretizations for varying applications such as $[22,13,16]$.

\section{Acknowledgements}

The author also wishes to acknowledge Sage open-source mathematical software system, which is a free, GPL-licensed, open source codebase mainly written in Python. 


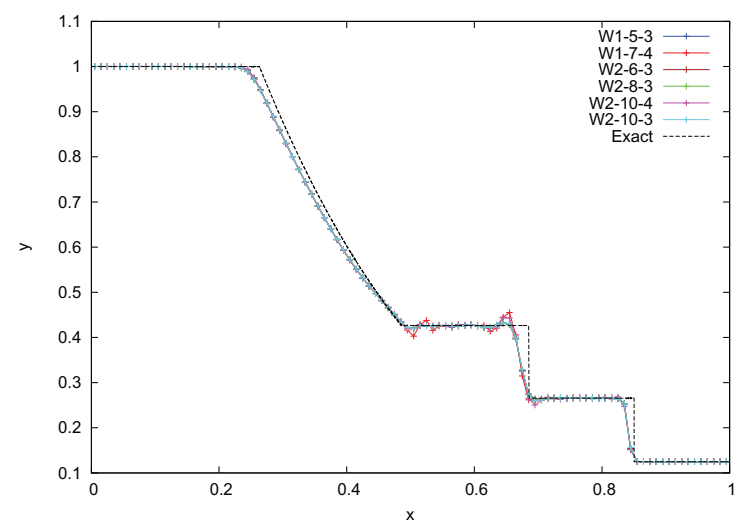

(a) Full Plot

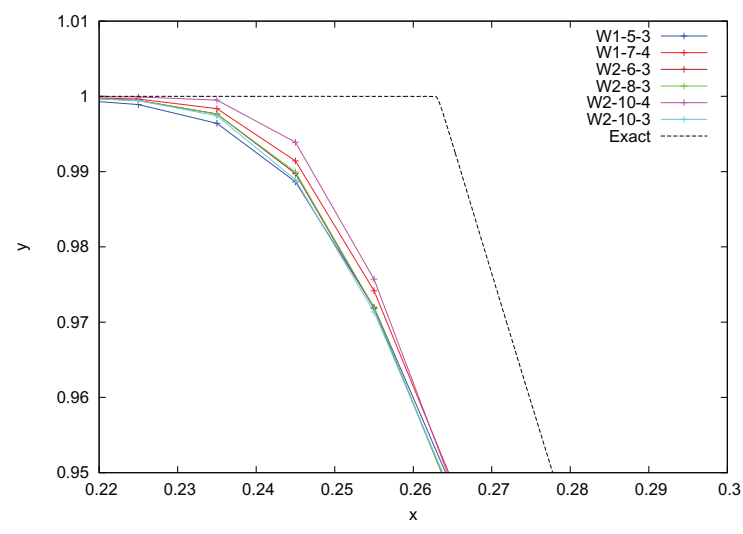

(b) Zoomed at the top of the rarefaction

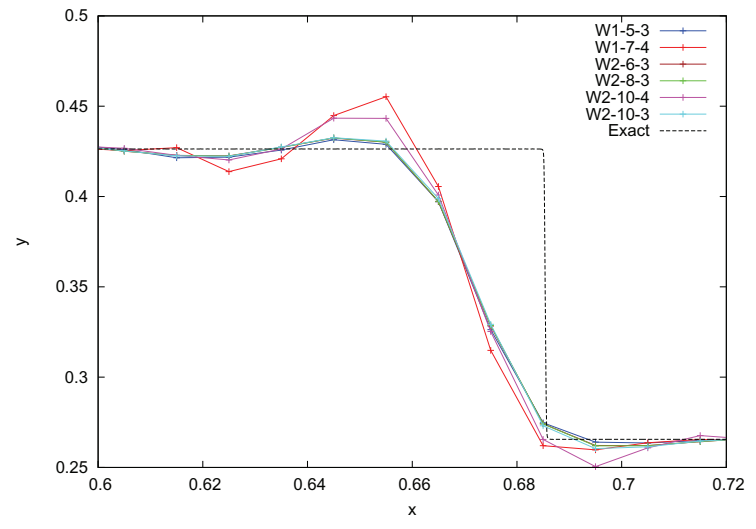

(d) Zoomed at the contact discontinuity

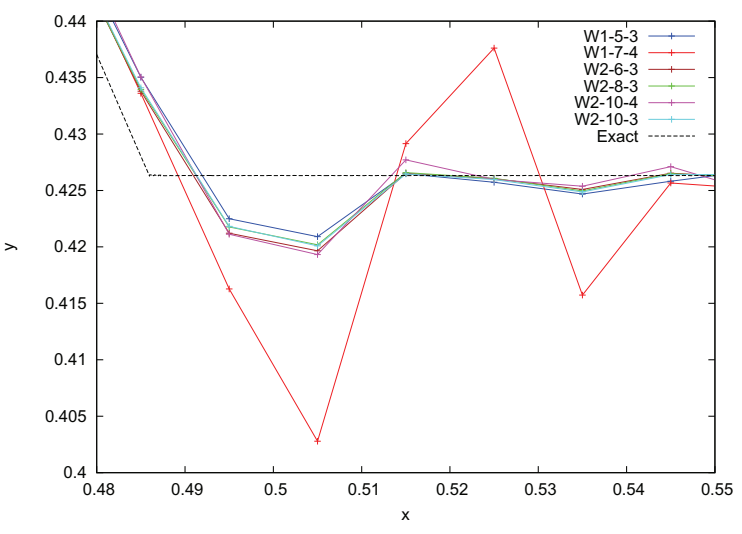

(c) Zoomed at the bottom of the rarefaction

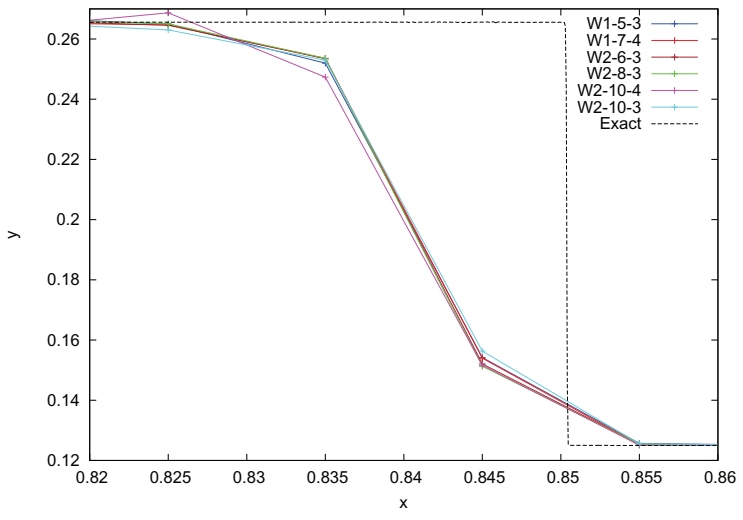

(e) Zoomed at the shock

Figure 3: Plots of numerical solutions of density for the Sod shock tube problem using 100 cells. 


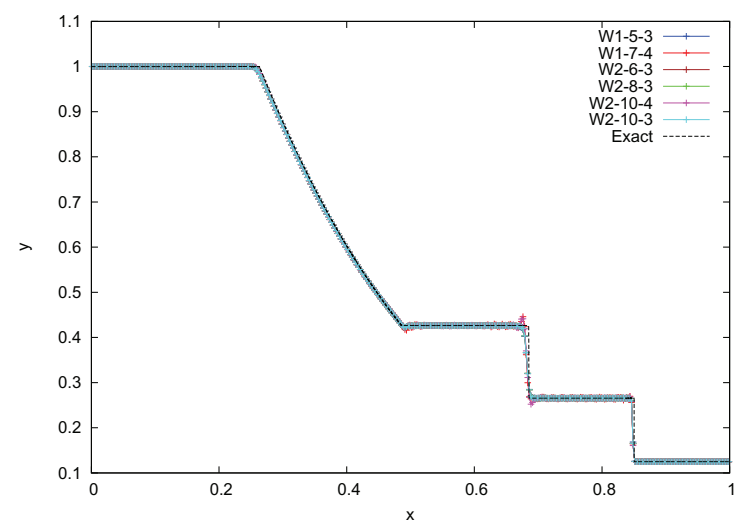

(a) Full Plot

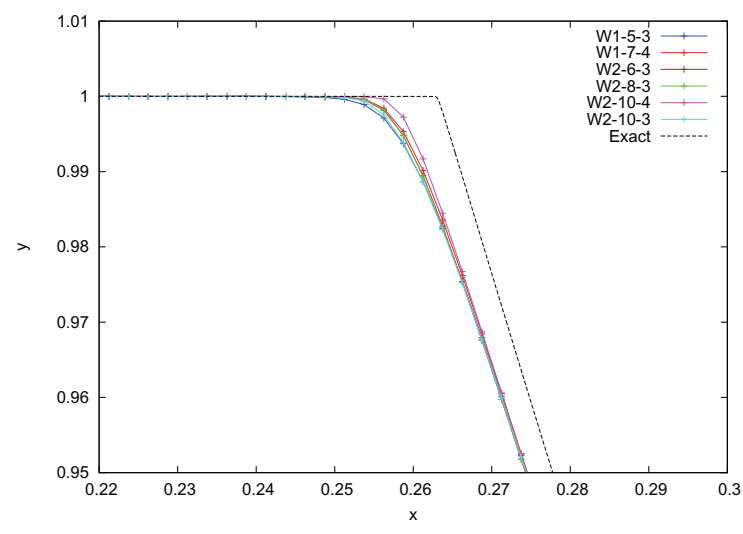

(b) Zoomed at the top of the rarefaction

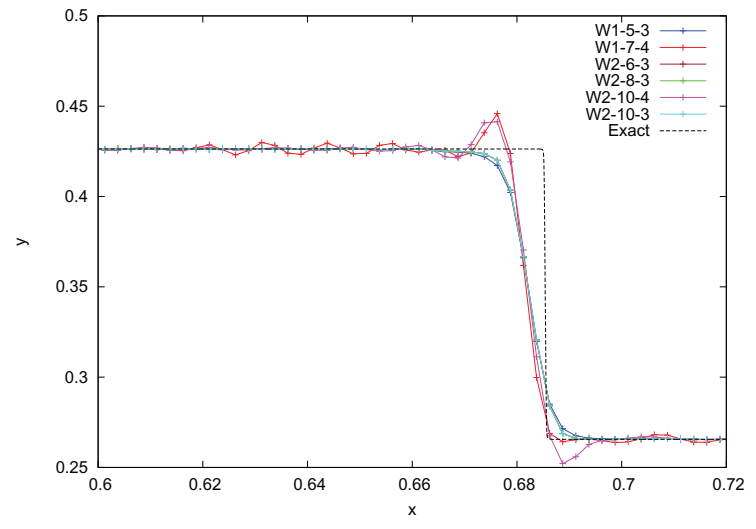

(d) Zoomed at the contact discontinuity

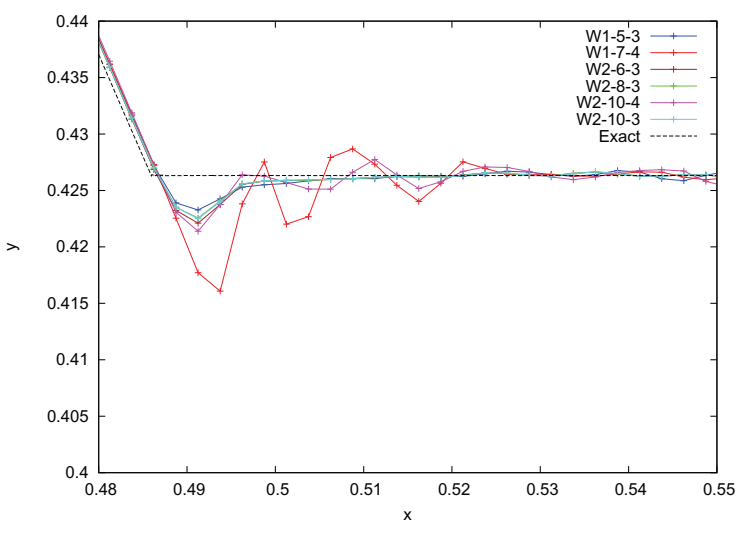

(c) Zoomed at the bottom of the rarefaction

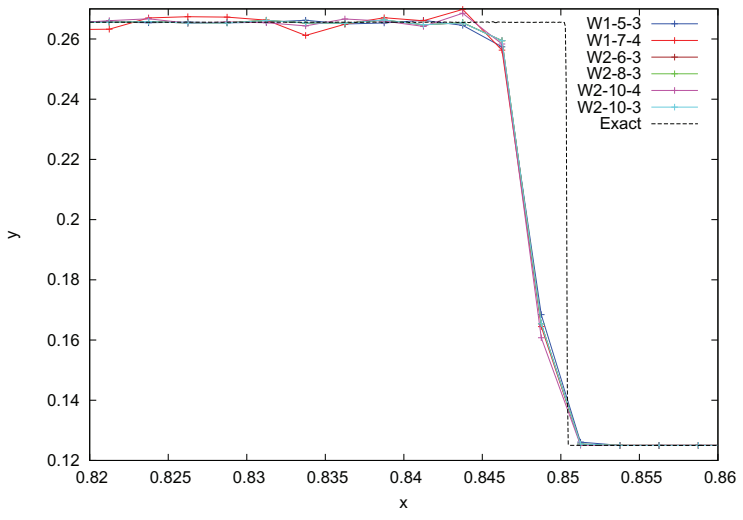

(e) Zoomed at the shock

Figure 4: Plots of numerical solutions of density for the Sod shock tube problem using 400 cells. 
This research used resources of the National Center for Computational Sciences at Oak Ridge National Laboratory, which is supported by the Office of Science of the U.S. Department of Energy under Contract No. DE-AC05-00OR22725.

[1] G.-S. Jiang, C.-W. Shu, Efficient implementation of weighted ENO schemes, Journal of computational physics 126 (1) (1996) 202-228.

[2] X.-D. Liu, S. Osher, T. Chan, Weighted essentially non-oscillatory schemes, Journal of computational physics 115 (1) (1994) 200-212.

[3] G. Capdeville, A hermite upwind WENO scheme for solving hyperbolic conservation laws, Journal of Computational Physics 227 (4) (2008) 2430-2454.

[4] J. Qiu, C.-W. Shu, Hermite WENO schemes and their application as limiters for Runge-Kutta discontinuous galerkin method: one-dimensional case, Journal of Computational Physics 193 (1) (2004) $115-135$.

[5] J. Qiu, C.-W. Shu, Hermite WENO schemes for Hamilton-Jacobi equations, Journal of Computational Physics 204 (1) (2005) 82-99.

[6] J. Qiu, Hermite WENO schemes with Lax-Wendroff type time discretizations for Hamilton-Jacobi equations, Journal of Computational Mathematics 25 (2).

[7] M. R. Norman, Algorithmic improvements for schemes using the ADER time discretization, Journal of Computational Physics 243 (15) (2013) 176-178.

[8] M. R. Norman, H. Finkel, Multi-moment ADER-Taylor methods for systems of conservation laws with source terms in one dimension, Journal of Computational Physics 231 (20) (2012) 6622-6642.

[9] M. R. Norman, R. D. Nair, F. H. Semazzi, A low communication and large time step explicit finitevolume solver for non-hydrostatic atmospheric dynamics, Journal of Computational Physics 230 (4) (2011) 1567-1584.

[10] R. J. LeVeque, Finite Volume Methods for Hyperbolic Problems, Cambridge University Press, 2002.

[11] M. R. Norman, A weno-limited, ader-dt, finite-volume scheme for efficient, robust, and communicationavoiding multi-dimensional transport, Journal of Computational Physics (2014) under review.

[12] V. A. Titarev, E. F. Toro, ADER schemes for three-dimensional non-linear hyperbolic systems, Journal of Computational Physics 204 (2) (2005) 715-736.

[13] E. F. Toro, V. A. Titarev, ADER schemes for scalar non-linear hyperbolic conservation laws with source terms in three-space dimensions, Journal of Computational Physics 202 (1) (2005) 196-215.

[14] E. F. Toro, M. Dumbser, ADER schemes for time-dependent PDEs: review and applications to astrophysics, in: Numerical Modeling of Space Plasma Flows, Astronum-2009, Vol. 429, 2010, p. 281.

[15] E. F. Toro, V. A. Titarev, Derivative riemann solvers for systems of conservation laws and ADER methods, Journal of Computational Physics 212 (1) (2006) 150-165.

[16] M. Dumbser, D. S. Balsara, E. F. Toro, C.-D. Munz, A unified framework for the construction of one-step finite volume and discontinuous galerkin schemes on unstructured meshes, Journal of Computational Physics 227 (18) (2008) 8209-8253.

[17] J. Shi, C. Hu, C.-W. Shu, A technique of treating negative weights in WENO schemes, Journal of Computational Physics 175 (1) (2002) 108-127.

[18] J.-B. Cheng, E. F. Toro, S. Jiang, W. Tang, A sub-cell WENO reconstruction method for spatial derivatives in the ADER scheme, Journal of Computational Physics 251 (2013) 53-80. 
[19] A. K. Henrick, T. D. Aslam, J. M. Powers, Mapped weighted essentially non-oscillatory schemes: achieving optimal order near critical points, Journal of Computational Physics 207 (2) (2005) 542-567.

[20] D. S. Balsara, C. Meyer, M. Dumbser, H. Du, Z. Xu, Efficient implementation of ADER schemes for euler and magnetohydrodynamical flows on structured meshes-Speed comparisons with Runge-Kutta methods, Journal of Computational Physics 235 (2013) 934-969.

[21] D. S. Balsara, T. Rumpf, M. Dumbser, C.-D. Munz, Efficient, high accuracy ADER-WENO schemes for hydrodynamics and divergence-free magnetohydrodynamics, J. Comput. Phys. 228 (7) (2009) 24802516. doi:10.1016/j.jcp.2008.12.003.

URL http://dx.doi.org/10.1016/j.jcp.2008.12.003

[22] M. Dumbser, C. Enaux, E. F. Toro, Finite volume schemes of very high order of accuracy for stiff hyperbolic balance laws, Journal of Computational Physics 227 (8) (2008) 3971-4001. doi:10.1016/j.jcp.2007.12.005.

URL http://www.sciencedirect.com/science/article/pii/S0021999107005578 